\title{
Quintessence as a runaway dilaton
}

\author{
M. Gasperini \\ Dipartimento di Fisica, Università di Bari, Via G. Amendola 173, 70126 Bari, Italy \\ and Istituto Nazionale di Fisica Nucleare, Sezione di Bari, Bari, Italy \\ F. Piazza \\ Dipartimento di Fisica, Università di Milano Bicocca, Piazza delle Scienze 3, I-20126 Milan, Italy \\ and Laboratoire de Physique Théorique, Université Paris Sud, 91405 Orsay, France \\ G. Veneziano \\ Laboratoire de Physique Théorique, Université Paris Sud, 91405 Orsay, France \\ and Theoretical Physics Division, CERN, CH-1211 Geneva 23, Switzerland
}

(Received 4 August 2001; published 21 December 2001)

\begin{abstract}
We consider a late-time cosmological model based on a recent proposal that the infinite-bare-coupling limit of superstring or $\mathrm{M}$ theory exists and has good phenomenological properties, including a vanishing cosmological constant, and a massless, decoupled dilaton. As it runs away to $+\infty$, the dilaton can play the role of the quintessence field recently advocated to drive the late-time accelerated expansion of the Universe. If, as suggested by some string theory examples, appreciable deviations from general relativity persist even today in the dark matter sector, the Universe may smoothly evolve from an initial "focusing" stage, lasting until radiation-matter equality, to a "dragging" regime, which eventually gives rise to an accelerated expansion with frozen $\Omega$ (dark energy) $/ \Omega$ (dark matter).
\end{abstract}

DOI: 10.1103/PhysRevD.65.023508 PACS number(s): 98.80.Cq, 04.50. $+\mathrm{h}, 11.25 . \mathrm{Mj}, 95.35 .+\mathrm{d}$

\section{INTRODUCTION}

According to recent astrophysical observations, our Universe, since a redshift of $\mathcal{O}(1)$, appears to have undergone a phase of accelerated expansion [1,2]. This result can be combined with the recent estimates of the average mass density of the Universe [3], $\Omega_{m} \simeq 0.3-0.4$ (in critical units), and with recent measurements of the cosmic microwave background (CMB) anisotropy peaks [4], pointing at a nearly critical total energy density, $\Omega_{T} \simeq 1$. One is then led to the conclusion that the present cosmological evolution, when described in terms of an effective fluid entering Einstein's equations, should be (marginally) dominated by a "dark energy" component $\rho_{x}$ characterized by a (sufficiently) negative effective pressure, $p_{x}<-\rho_{x} / 3$.

The simplest candidate for such a missing energy is a positive cosmological constant $\Lambda$, of order $H_{0}^{2}$. Such an identification, however, unavoidably raises a series of difficult questions. In particular, (a) why is $\Lambda$ so small in particle physics units? Explaining a finite but very small value for $\Lambda$ may turn out to be even harder than finding a reason why it is exactly zero. This is the so-called fine-tuning problem for $\Lambda$, see for instance [5]. (b) Why is $\Lambda \sim \rho_{m 0}$, where $\rho_{m 0}$ is the present value (in Planck units) of the (dark) matter energy density? This is the so-called "cosmic coincidence" problem [6].

At present, the most promising scenarios for solving (at least part of) the above problems introduce a single scalar field, dubbed "quintessence" [7], whose potential goes to zero asymptotically (leaving therefore just the usual puzzle of why the "true" cosmological constant vanishes). The scalar field slowly rolls down such a potential reaching infinity (and zero energy) only after an infinite (or very long) time.
While doing so, quintessence produces an effective, timedependent, cosmic energy density $\rho_{x}$ accompanied by a sufficiently negative pressure, i.e. a sort of effective cosmological constant. By making $\Lambda_{e f f} \sim H^{2}$ time dependent, this can naturally explain the smallness of the present effective vacuum energy density. However, if, as in general relativity, dust energy and an effective cosmological constant have different time dependence, it can hardly explain why $\Lambda \sim \rho_{m 0}$. For a recent review of the relative merits of a cosmological constant and quintessence, see Ref. [8].

As far as identifying quintessence is concerned, the inflaton itself could be a candidate [9]. But also more exotic possibilities have been considered, in particular some motivated by the wish to solve the above-mentioned cosmic coincidence problem $[10,11]$. In any case, as is the case for the inflaton, the quintessence field does not have, as yet, an obvious place in any fundamental theory of elementary particles. One should also mention, at this point, that, if quintessence may help with the problems typical of the cosmological constant interpretation, it is likely to create a new one of its own: in order to play its role, the quintessence field must be extremely light and can thus mediate a new long-range (of order $\mathrm{H}_{0}^{-1}$ ) force, which is strongly constrained observationally. This is an important constraint to be imposed on any specific scalar field model of quintessence, either minimally or non-minimally [12] coupled to gravity.

At first sight, the search for a quintessence candidate in particle physics looks easier than the one for an inflaton. For instance, fundamental or effective scalar fields with potentials running to zero at infinity are ubiquitous in supersymmetric field theories and/or in string/M theory. They are usually referred to as moduli fields since, in perturbation theory, they parametrize the space of inequivalent vacua and corre- 
spond to exactly flat directions (equivalently, to exactly massless fields). Non-perturbative effects (e.g. gauge-theory instantons) are expected to lift these flat directions, just preserving those that correspond to small or vanishing coupling. Examples are the run-away vacua of supersymmetric gauge theories (see, for instance, [13] for quintessence models based on the latter possibility), or the dilaton modulus $\phi$ in the limit $\phi \rightarrow-\infty$.

However, if we were to take one of these moduli as quintessence, we would immediately run into the problem that the acceleration of the Universe should be accompanied by a drift of interactions towards triviality. For Newton's constant, and even more so for the fine-structure constant, this kind of time variation is very strongly constrained. Furthermore, typical couplings of moduli fields to ordinary matter are of gravitational order, and this creates the already mentioned problem of new, unwanted long-range forces. For all these reasons the conventional attitude towards moduli fields has been (see e.g. [14]) to postulate that they develop nonperturbative potentials, providing them with both a mass and a freezing mechanism (see however [15] for an alternative that is closer, in spirit, to the one advocated here).

Another possible problem with the identification of a string modulus with quintessence is that we would like to freeze the moduli at values that provide the correct values of the coupling constant and unification scale of grand unified theories (GUTs). For instance, the dilaton and compactification volume $V_{6}$ should be frozen at values such that [16]

$$
\alpha_{G U T}^{-1} \sim\left(M_{P} / M_{s}\right)^{2} \sim e^{-\phi}, \quad M_{P} / M_{G U T} \sim \alpha_{G U T}^{-2 / 3} g_{s}^{1 / 6},
$$

where $M_{P}, M_{s}$ and $M_{G U T}$ are the Planck, string and GUT scales, $g_{s}=e^{\phi} V_{6} M_{s}^{6}$ is the string coupling, and $e^{\phi}$ is the tree-level effective four-dimensional coupling (thus, in more standard string-theory notation [17], our dilaton is related to the real part of the $S$ modulus by $\operatorname{Re}\{S\}=e^{-\phi}$ ).

Unfortunately, it looks unlikely that non-perturbative effects will be significant enough in this region to stabilize the moduli. Also, perturbative unification gives too low a value for $M_{P} / M_{G U T}[16,18]$. In this respect, the situation can be drastically improved by considering the $M$-theory limit, $g_{s}$ $\rightarrow \infty$, while still keeping the four-dimensional effective couplings perturbative $(S \gg 1)$ [18]. Even then, the moduli would presumably freeze out in a typical (and cosmologically tiny) particle-physics time, and therefore cannot implement the conventional, slow-roll quintessential scenario. In spite of these difficulties, unconventional models of quintessence based on the stabilization of the dilaton in the perturbative regime are not completely excluded, as recently discussed by one of us [19].

There is, however, another possibility for making the dilaton a candidate for quintessence. As we have already mentioned, the region of large negative $\phi$ corresponds to the trivial vacuum. The idea that the Universe may have started, long before the big bang, in this region is actually the basis of the so-called pre-big-bang scenario in string cosmology (for recent reviews see [20]). Here we are asking instead whether the dilaton can play the role of quintessence at very late times (such as today), not by evolving towards $-\infty$ and triviality, but by going towards $+\infty$ and strong coupling. Such a proposal looks absurd at first since, if we do not see a drift towards zero coupling, we do not experience one towards increasing strength either. In order for this idea to make sense we have to assume that the strong-coupling limit of string or M theory exists, is smooth, and resembles our world. Can this make sense at all?

It has been argued by one of us [21] that the answer to the above question can be affirmative, if we assume a certain structure of the quantum loop corrections to the string effective action suggested by large- $N$ counting arguments. In the strong coupling limit (which could either be the self-dual value $S=1$ or, if $S$-duality is broken, $\phi \rightarrow+\infty$ ) gravitational and gauge coupling would be determined entirely by loop corrections (as in the so-called induced-gravity/gauge idea [22]), and would "saturate" at "small" values because of the large number of fields entering the loops (e.g. the large number $N$ of gauge bosons, or the large value of the quadratic Casimir $C_{A}$, for gauge groups like $E_{8}$ ). Typically, Eq. (1.1) would be replaced (at $\phi \gg 1$ ) by

$$
\alpha_{G U T}^{-1} \sim C_{A}+\mathcal{O}\left(e^{-\phi}\right), \quad\left(M_{P} / M_{s}\right)^{2} \sim N+\mathcal{O}\left(e^{-\phi}\right) .
$$

In this picture there is naturally an asymptotic decoupling mechanism of ordinary matter to the dilaton, whose effective mass goes to zero at late times. The problem remains, of course, of explaining why the cosmological constant vanishes in superstring/M theory, not only at zero coupling where supersymmetry protects it, but also at infinite (bare) coupling. Possibly, some new, stringy symmetry can explain this. It will simply be assumed to be the case in this paper.

As the dilaton is non-universally coupled to different types of matter fields, its coupling to ordinary matter can be asymptotically tiny (as to satisfy constraints from gravitational experiments [15]), and much stronger (as first suggested in [23]) to typical dark matter candidates, such as the axion. In that case, the dilaton to dark-matter coupling leads to an initial evolution, which is similar to the "tracking" regime [24] of conventional models of quintessence, but takes place before potential energy becomes appreciable. Later on, the interplay of the dark-matter dilatonic charge and of the dilaton potential leads to an accelerated expansion in which the relative fraction of dark energy and dark matter remains fixed (and of order 1), thus offering a possible explanation of the cosmic coincidence, as we will illustrate through explicit examples.

The paper is organized as follows. In Sec. II we present the effective string cosmology equations, in the small curvature-but arbitrary coupling-regime, with generic matter sources non-minimally coupled to the dilaton. In Sec. III we discuss analytically a possible late-time attractor characterized by a constant positive acceleration and a fixed ratio of dark matter and dark energy. In Sec. IV we provide a semi-quantitative description of the previous phase, during which the dilaton potential can be neglected. This phase is characterized by a "focusing" of the energy densities of the various components of the cosmological fluid (which occurs 
before the epoch of matter-radiation equilibrium), and by a subsequent "dragging" regime in which the dilaton energy density tends to follow that of non-relativistic (dark) matter. We also discuss here the main phenomenological constraints that have to be imposed on the scenario. In Sec. V we consider a typical example of a string cosmology model including radiation, baryonic and cold dark matter, and we present the results of explicit numerical integrations. Our conclusions are summarized in Sec. VI.

\section{COSMOLOGICAL EQUATIONS IN THE STRING AND EINSTEIN FRAMES}

Our starting point is the string-frame, low-energy, gravidilaton effective action [17], to lowest order in the $\alpha^{\prime}$ expansion, but including dilaton-dependent loop (and nonperturbative) corrections, encoded in a few "form factors" $\psi(\phi), Z(\phi), \alpha(\phi), \ldots$, and in an effective dilaton potential $V(\phi)$ (see also [15]). In the formula,

$$
\begin{aligned}
S= & -\frac{M_{s}^{2}}{2} \int d^{4} x \sqrt{-\tilde{g}}\left[e^{-\psi(\phi)} \widetilde{R}+Z(\phi)(\widetilde{\nabla} \phi)^{2}+\frac{2}{M_{s}^{2}} V(\phi)\right] \\
& -\frac{1}{16 \pi} \int d^{4} x \frac{\sqrt{-\tilde{g}}}{\alpha(\phi)} F_{\mu \nu}^{2}+\Gamma_{m}(\phi, \tilde{g}, \text { matter })
\end{aligned}
$$

[conventions: metric signature: $(+,-,-,-), R_{\mu \nu \alpha} \beta$ $\left.=\partial_{\mu} \Gamma_{\nu \alpha}{ }^{\beta}-\ldots, R_{\mu \nu}=R_{\alpha \mu \nu}{ }^{\alpha}\right]$. Here $M_{s}^{-1}=\lambda_{s}$ is the fundamental string-length parameter, $\tilde{g}$ is the sigma-model metric minimally coupled to fundamental strings, $\widetilde{R}, \widetilde{\nabla}$ are the curvature and the covariant derivative referred to $\tilde{g}$, and $F_{\mu \nu}$ is the gauge field of some fundamental (GUT) group $[\alpha(\phi)$ is the corresponding gauge coupling]. We imagine having already compactified 6 dimensions and having frozen the corresponding moduli at the string scale. Following the basic proposal made in [21], we shall assume that the form factors $\psi(\phi), Z(\phi), \alpha(\phi)$ approach a finite, physically interesting limit as $\phi \rightarrow+\infty$ while, in the same limit, $V \rightarrow 0$.

The fields appearing in the matter action $\Gamma_{m}$ are in general non-minimally and non-universally coupled to the dilaton (also because of the loop corrections [14]). Their gravitational and dilatonic "charge densities," $\widetilde{T}_{\mu \nu}$ and $\tilde{\sigma}$, are defined as follows:

$$
\frac{\delta \Gamma}{\delta \widetilde{g}^{\mu \nu}}=\frac{1}{2} \sqrt{-\tilde{g}} \widetilde{T}_{\mu \nu}, \quad \frac{\delta \Gamma}{\delta \phi}=-\frac{1}{2} \sqrt{-\tilde{g}} \tilde{\sigma},
$$

and it is important to stress that, when $\tilde{\sigma} \neq 0$, the gravidilaton effective theory is radically different from a typical, JordanBrans-Dicke type model of scalar-tensor gravity [25]. We shall give a prototype form of $\Gamma_{m}$ in the following section, after passing to the Einstein frame.

The variation of Eq. (2.1) with respect to $\tilde{g}_{\mu \nu}$ then gives the equations

$$
\begin{aligned}
\widetilde{G}_{\mu \nu} & +\psi^{\prime} \widetilde{\nabla}_{\mu} \widetilde{\nabla}_{\nu} \phi+\left[e^{\psi} Z-\psi^{\prime 2}+\psi^{\prime \prime}\right] \widetilde{\nabla}_{\mu} \phi \widetilde{\nabla}_{\nu} \phi \\
& +\frac{1}{2} \widetilde{g}_{\mu \nu}\left[\left(2 \psi^{\prime 2}-2 \psi^{\prime \prime}-e^{\psi} Z\right)(\widetilde{\nabla} \phi)^{2}-2 \psi^{\prime}\left(\widetilde{\nabla}^{2} \phi\right)\right. \\
& \left.-e^{\psi} V(\phi)\right]=\lambda_{s}^{2} e^{\psi} \widetilde{T}_{\mu \nu},
\end{aligned}
$$

where $\widetilde{G}_{\mu \nu}$ is the Einstein tensor, and a prime denotes differentiation with respect to $\phi$. The variation with respect to $\phi$, using the trace of Eq. (2.3) to eliminate $\widetilde{R}$, leads to the equation

$$
\begin{gathered}
\left(3 \psi^{\prime 2}-2 e^{\psi} Z\right)\left(\tilde{\nabla}^{2} \phi\right)+\left[e^{\psi}\left(Z \psi^{\prime}-Z^{\prime}\right)+\psi^{\prime}\left(3 \psi^{\prime \prime}-3 \psi^{\prime 2}\right)\right] \\
\times(\widetilde{\nabla} \phi)^{2}+e^{\psi}\left(2 \psi^{\prime} V+V^{\prime}\right)+\lambda_{s}^{2} e^{\psi}\left(\psi^{\prime} \widetilde{T}+\widetilde{\sigma}\right)=0 .
\end{gathered}
$$

We shall assume an isotropic, spatially flat metric background (appropriate to the present cosmological configuration), and a perfect fluid model of source. In the cosmic-time gauge we thus set

$$
\begin{aligned}
\tilde{g}_{\mu \nu} & =\operatorname{diag}\left(1,-\tilde{a}^{2}(\tilde{t}) \delta_{i j}\right), \quad \widetilde{T}_{\mu}^{\nu}=\operatorname{diag}\left(\tilde{\rho},-\tilde{p} \delta_{i}^{j}\right), \\
\phi & =\phi(\tilde{t}), \quad \tilde{\sigma}=\tilde{\sigma}(\tilde{t}),
\end{aligned}
$$

and one can easily check, combining the above equations, that the matter stress tensor is not covariantly conserved (even in this frame), but satisfies the equation

$$
\dot{\tilde{\rho}}+3 \widetilde{H}(\tilde{\rho}+\tilde{p})=\frac{\tilde{\sigma}}{2} \dot{\phi} .
$$

For the purpose of this paper, and for an easier comparison with previous discussions of the quintessential scenario, it is however convenient to represent the dynamical evolution of the background in the more conventional Einstein frame, characterized by a metric $g_{\mu \nu}$ minimally coupled to the dilaton, and defined by the conformal transformation $\tilde{g}_{\mu \nu}=c_{1}^{2} g_{\mu \nu} e^{\psi}$. Here $c_{1}^{2}$ parametrizes the asymptotic behavior of $\psi(\phi)$,

$$
c_{1}^{2}=\lim _{\phi \rightarrow+\infty} \exp \{-\psi(\phi)\},
$$

and thus controls the asymptotic ratio between the string and the Planck scale, $M_{P}^{2}=c_{1}^{2} M_{s}^{2}$. In the Einstein frame the action (2.1) becomes

$$
\begin{aligned}
S= & -\frac{M_{P}^{2}}{2} \int d^{4} x \sqrt{-g}\left[R-\frac{k(\phi)^{2}}{2}(\nabla \phi)^{2}+\frac{2}{M_{P}^{2}} \hat{V}(\phi)\right] \\
& -\frac{1}{16 \pi} \int d^{4} x \frac{\sqrt{-g}}{\alpha(\phi)} F_{\mu \nu}^{2}+\Gamma_{m}\left(\phi, c_{1}^{2} g_{\mu \nu} e^{\psi}, \text { matter }\right),
\end{aligned}
$$

where we have defined

$$
k^{2}(\phi)=3 \psi^{\prime 2}-2 e^{\psi} Z, \quad \hat{V}=c_{1}^{4} e^{2 \psi} V .
$$


For later use it is also convenient to define a canonical dilaton field by

$$
d \hat{\phi}=\frac{M_{P}}{\sqrt{2}} k(\phi) d \phi,
$$

although, in solving the equations, it will be easier to work directly with the original field $\phi$.

We now choose, also in the Einstein frame, the cosmictime gauge, according to the rescaling

$$
\begin{aligned}
& \tilde{a}=c_{1} a e^{\psi / 2}, \quad d \tilde{t}=c_{1} d t e^{\psi / 2}, \quad \rho=c_{1}^{2} e^{2 \psi} \tilde{\rho}, \\
& p=c_{1}^{2} e^{2 \psi} \tilde{p}, \quad \sigma=c_{1}^{2} e^{2 \psi} \tilde{\sigma} .
\end{aligned}
$$

From the $(0,0)$ and $(i, j)$ components of Eq. (2.3) we obtain, respectively, the Einstein cosmological equations [in units such that $\left.M_{P}^{2}=c_{1}^{2} M_{s}^{2} \equiv(8 \pi G)^{-1}=2\right]$

$$
\begin{aligned}
6 H^{2} & =\rho+\rho_{\phi}, \\
4 \dot{H}+6 H^{2} & =-p-p_{\phi},
\end{aligned}
$$

while from the dilaton equation (2.4) we get

$$
\begin{gathered}
k^{2}(\phi)(\ddot{\phi}+3 H \dot{\phi})+k(\phi) k^{\prime}(\phi) \dot{\phi}^{2}+\hat{V}^{\prime}(\phi) \\
+\frac{1}{2}\left[\psi^{\prime}(\phi)(\rho-3 p)+\sigma\right]=0 .
\end{gathered}
$$

In the above equations $H=\dot{a} / a$, a dot denotes differentiation with respect to the Einstein cosmic time, and we have used the definitions

$$
\rho_{\phi}=\frac{1}{2} k^{2}(\phi) \dot{\phi}^{2}+\hat{V}(\phi), \quad p_{\phi}=\frac{1}{2} k^{2}(\phi) \dot{\phi}^{2}-\hat{V}(\phi) .
$$

The combination of Eqs. (2.12)-(2.14) leads finally to the coupled conservation equations for the matter and dilaton energy density, respectively:

$$
\begin{array}{r}
\dot{\rho}+3 H(\rho+p)-\frac{1}{2} \dot{\phi}\left[\psi^{\prime}(\phi)(\rho-3 p)+\sigma\right]=0, \\
\dot{\rho}_{\phi}+3 H\left(\rho_{\phi}+p_{\phi}\right)+\frac{1}{2} \dot{\phi}\left[\psi^{\prime}(\phi)(\rho-3 p)+\sigma\right]=0 .
\end{array}
$$

For further applications, and for a more transparent numerical integration, it is also convenient to parametrize the time evolution of all variables in terms of the logarithm of the scale factor, $\chi=\ln \left(a / a_{i}\right)$, where $a_{i}$ corresponds to the initial scale, ${ }^{1}$ and to separate the radiation, baryonic and nonbaryonic matter components of the cosmological fluid by setting

\footnotetext{
${ }^{1}$ The relation between $\chi$ and the redshift $z$ is $\chi=-\ln (1+z)$ $+\ln \left(a_{0} / a_{i}\right)$, where $a_{0}$ is the present value of the scale factor.
}

$$
\begin{aligned}
& \rho=\rho_{r}+\rho_{b}+\rho_{d} \equiv \rho_{r}+\rho_{m}, \quad p=\frac{1}{3} \rho_{r}, \\
& \sigma=\sigma_{r}+\sigma_{b}+\sigma_{d} \equiv \sigma_{r}+\sigma_{m} .
\end{aligned}
$$

The dilaton equation and the Einstein equation (2.12) can then be written, respectively, as

$$
\begin{aligned}
2 H^{2} k^{2} & \frac{d^{2} \phi}{d \chi^{2}}+k^{2}\left(\frac{1}{2} \rho_{m}+\frac{1}{3} \rho_{r}+\hat{V}\right) \frac{d \phi}{d \chi}+2 H^{2} k k^{\prime}\left(\frac{d \phi}{d \chi}\right)^{2} \\
+ & 2 \hat{V}^{\prime}+\psi^{\prime} \rho_{m}+\sigma=0 \\
H^{2}\left[6-\frac{k^{2}}{2}\left(\frac{d \phi}{d \chi}\right)^{2}\right] & =\rho_{m}+\rho_{r}+\hat{V}
\end{aligned}
$$

The matter evolution equation (2.16) can be split into the various components as

$$
\begin{array}{r}
\frac{d \rho_{r}}{d \chi}+4 \rho_{r}-\frac{\sigma_{r}}{2} \frac{d \phi}{d \chi}=0, \\
\frac{d \rho_{b}}{d \chi}+3 \rho_{b}-\frac{1}{2}\left(\psi^{\prime} \rho_{b}+\sigma_{b}\right) \frac{d \phi}{d \chi}=0, \\
\frac{d \rho_{d}}{d \chi}+3 \rho_{d}-\frac{1}{2}\left(\psi^{\prime} \rho_{d}+\sigma_{d}\right) \frac{d \phi}{d \chi}=0 .
\end{array}
$$

Finally, Eq. (2.19) is also equivalent to the dilaton conservation equation (2.17), which becomes

$$
\frac{d \rho_{\phi}}{d \chi}+6 \rho_{\phi}-6 \hat{V}(\phi)+\frac{1}{2}\left(\psi^{\prime} \rho_{m}+\sigma\right) \frac{d \phi}{d \chi}=0 .
$$

\section{ACCELERATED LATE-TIME ATTRACTORS WITH CONSTANT $\Omega_{\phi}$}

As a first step towards a "dilatonic" interpretation of quintessence we will now discuss the possibility that the above equations, together with a string-theory motivated potential and loop corrections, are asymptotically solved by an accelerated expansion, $\ddot{a}>0$, with frozen ratio $\rho_{m} / \rho_{\phi}$ of the order of unity. This last property, in particular, is expected to solve (or at least alleviate) the cosmic coincidence problem $[10,11]$.

Under the assumption made in [21] that the form factors appearing in Eq. (2.1) have a finite limit as $\phi \rightarrow+\infty$, and assuming the validity of an asymptotic Taylor expansion, we write:

$$
\begin{gathered}
e^{-\psi(\phi)}=c_{1}^{2}+b_{1} e^{-\phi}+\mathcal{O}\left(e^{-2 \phi}\right), \\
Z(\phi)=-c_{2}^{2}+b_{2} e^{-\phi}+\mathcal{O}\left(e^{-2 \phi}\right), \\
\alpha(\phi)^{-1}=\alpha_{0}^{-1}+b e^{-\phi}+\mathcal{O}\left(e^{-2 \phi}\right),
\end{gathered}
$$

where $c_{1}^{2}, c_{2}^{2}$ are assumed to be of the same order [typically of order $10^{2}$ since, as already noted, $\left.c_{1}^{2}=\left(M_{P} / M_{s}\right)^{2}\right]$, and 
$\alpha_{0}$ is to be identified with the unified gauge coupling at the GUT scale, i.e. $\alpha_{0} \simeq 1 / 25$. Unlike the model discussed in [26] our model thus describes, in the strong coupling limit $\phi \rightarrow$ $+\infty$, a minimally coupled, canonical scalar field $\hat{\phi}$ $=\sqrt{2}\left(c_{2} / c_{1}\right) \phi$, see Eq. (2.10). In the opposite limit, $\phi \rightarrow$ $-\infty$, the gravi-dilaton string effective action reduces, as usual, to an effective Brans-Dicke model with parameter $\omega$ $=-1$. We note that it is not hard to chose $\psi(\phi)$ and $Z(\phi)$ in such a way that the kinetic term of the dilaton keeps the correct sign at all values of $\phi$ (see the example given in Sec. V).

Similarly, the assumption that $V$ originates from nonperturbative effects, and that $V \rightarrow 0$ as $\phi \rightarrow \infty$, allows us to write, quite generically,

$$
\hat{V}(\phi)=V_{0} e^{-\phi}+\mathcal{O}\left(e^{-2 \phi}\right) .
$$

Since the overall normalization of the potential $V_{0}$ is nonperturbative, it should be related to the asymptotic value of the gauge coupling $\alpha_{0}$ by an expression of the form:

$$
V_{0}=M_{s}^{4} \exp \left(-\frac{4}{\beta \alpha_{0}}\right)=M_{*}^{4},
$$

with some model-dependent (one-loop) $\beta$-function coefficient $\beta$. For a comparison with earlier studies of an exponential potential $[27,28]$ we also note that, when referred to the canonically normalized dilaton field $\hat{\phi}$ defined in Eq. (2.10), the Einstein frame potential (3.2) asymptotically exibits an exponential behavior $\hat{V} \sim \exp \left(-\lambda \hat{\phi} / M_{P}\right)$, with $\lambda$ $=c_{1} / c_{2}=\sqrt{2} / k$ at $\phi \rightarrow+\infty$.

It is important to discuss the size of the potential needed for the viability of our scenario. Since the acceleration of the Universe appears to be a relatively recent phenomenon (even, possibly, an extremely recent one, as recently argued in [29]), the potential $V$ must enter the game very late, i.e. at an energy scale of the order of $\rho^{1 / 4} \sim 10^{-3} \mathrm{eV}$. Unless we want to play with an unnaturally large present value of $\phi$, this also fixes the scale of the potential in Eq. (3.2) as $V_{0}$ $\sim\left(10^{-3} \mathrm{eV}\right)^{4}$. As far as we know, this feature is common to all quintessence scenarios: the problem of an outstandingly small cosmological constant is traded for the introduction of another unnaturally small mass scale $M_{*}$.

In our context, we easily find that, in order to have a properly normalized potential, we need the constant $\beta$ appearing in the exponent of Eq. (3.3) to be somewhat smaller than the coefficient $\beta_{3}$ of the QCD beta function [see also the discussion after Eq. (3.5)], say $\beta \sim 0.6 \beta_{3}$. Given our ignorance of the origin of the dilaton potential, this looks perfectly acceptable, a priori. However, this apparent resolution of the fine-tuning problem should not hide the fact that the potential has to be adjusted very precisely if one wants to start the acceleration of the Universe not earlier than at redshift $z \sim \mathcal{O}(1)$, and not later than today. To the best of our knowledge there is no obvious explanation, at present, of this aspect of the coincidence problem.

Let us now come to the matter sector of the action (2.8). As a typical example of $\Gamma_{m}$ we take

$$
\begin{aligned}
\Gamma_{m}(\phi, g, \text { matter })= & \int d^{4} x \sqrt{-g} \bar{N}\left[i \not b+m_{N}(\phi)\right] N \\
& +\frac{1}{2} \int d^{4} x \sqrt{-g}\left[e^{\zeta(\phi)}\left(\partial_{\mu} D\right)^{2}\right. \\
& \left.-e^{\eta(\phi)} \mu^{2} D^{2}\right]
\end{aligned}
$$

the first term representing baryonic matter, the second (scalar) cold dark matter, while the gauge term appearing explicitly in Eq. (2.8) can already represent the radiation component of the cosmic fluid.

The non-observation of appreciable cosmological variations of the coupling constants [30], as well as the precision tests of Newtonian gravity [31] in the context of long-range dilatonic interactions, force us to assume that ordinary matter and radiation have nearly metric couplings to $\tilde{g}_{\mu \nu}$, i.e. that $\sigma_{b}, \sigma_{r} \simeq 0$ as $\phi \rightarrow \infty$. It is not hard to see how such a nearvanishing of dilatonic charges can be achieved starting from the actions (2.8),(3.4). Following Ref. [14] we have

$$
\frac{\sigma_{b}}{\rho_{b}} \sim \frac{\partial}{\partial \phi}\left(\ln \Lambda_{Q C D}\right), \quad \frac{\sigma_{r}}{\rho_{r}} \sim \frac{\partial}{\partial \phi}(\ln \alpha) .
$$

Given that $\Lambda_{Q C D} \sim M_{s} \exp \left(-1 / \beta_{3} \alpha\right)$ (with $\beta_{3}$ the coefficient of the QCD $\beta$-function), and using Eq. (3.1) for $\alpha$, it is clear that both $\sigma_{b}$ and $\sigma_{r}$ are exponentially suppressed at large, positive $\phi$. This decoupling mechanism is similar in spirit to the one proposed in [15], although it is supposed to occur at infinite bare coupling.

In the dark matter sector, on the contrary, we shall assume more generic quantum corrections. By taking for instance the action in Eq. (3.4), one has for the dilatonic charge of dark matter:

$$
\sigma_{d}=-\zeta^{\prime}(\phi) e^{\zeta(\phi)}\left(\partial_{\mu} D\right)^{2}+\eta^{\prime}(\phi) e^{\eta(\phi)} \mu^{2} D^{2} .
$$

Furthermore, the equations of motion for the $D$ field give a relation between the time-averaged quantities, $e^{\zeta(\phi)}\left\langle\dot{D}^{2}\right\rangle$ $=\mu^{2} e^{\eta(\phi)}\left\langle D^{2}\right\rangle$ (which is consistent with the interpretation of $D$ as non-relativistic matter, $p_{d}=0$, as assumed in the preceding section), and relate $\sigma_{d}$ and $\rho_{d}$ by a (generally $\phi$-dependent) proportionality factor

$$
\sigma_{d} / \rho_{d} \equiv q(\phi)=\eta^{\prime}(\phi)-\zeta^{\prime}(\phi) .
$$

The late-time behavior we will discuss takes place if we assume that, in the strong coupling limit (i.e., $\phi \gg 1), q(\phi)$ tends to a positive constant of order unity, and that the dark matter component dominates over baryonic matter and radiation. Thus, the regime we are considering is characterized [according to Eqs. (3.1),(3.2)] by

$$
\begin{gathered}
k^{2}(\phi)=2 c_{2}^{2} / c_{1}^{2}=2 / \lambda^{2}, \quad \sigma=\sigma_{d}, \quad \rho=\rho_{d}, \\
q(\phi)=q=\mathcal{O}(1), \quad \sigma_{d}=q \quad \rho_{d} .
\end{gathered}
$$

It follows that the dilaton coupling to the stress tensor can be asymptotically neglected with respect to the coupling to the dilatonic charge, as $\psi^{\prime} \simeq e^{-\phi} / c_{1}^{2} \ll 1$. The dilaton and dark 
matter conservation equations (2.23),(2.24) and the Einstein equations (2.12),(2.13) can then be written, asymptotically, in the form

$$
\begin{aligned}
\dot{\rho}_{d}+3 H \rho_{d}-\frac{q}{2} \rho_{m} \dot{\phi} & =0, \quad \dot{\rho}_{\phi}+6 H \rho_{k}+\frac{q}{2} \rho_{m} \dot{\phi}=0, \\
1 & =\Omega_{d}+\Omega_{k}+\Omega_{V}, \\
1+\frac{2 \dot{H}}{3 H^{2}} & =\Omega_{V}-\Omega_{k}
\end{aligned}
$$

where we have defined

$$
\begin{aligned}
& \rho_{d}=6 H^{2} \Omega_{d}, \quad \rho_{\phi}=\rho_{k}+\rho_{V}, \\
& \rho_{k}=6 H^{2} \Omega_{k}=\dot{\phi}^{2} / \lambda^{2}, \quad \rho_{V}=6 H^{2} \Omega_{V}=\hat{V} .
\end{aligned}
$$

We now look for solutions with asymptotically frozen dark-matter over dark-energy ratio, and frozen "equation of state." From the constraint (3.10) this is equivalent to the requirement that $\rho_{k}, \rho_{V}$ and $\rho_{d}$ scale in the same way, i.e.,

$$
\frac{d \log \rho_{\phi}}{d \chi}=\frac{d \log \rho_{d}}{d \chi}, \quad \frac{d \log \rho_{V}}{d \chi}=\frac{d \log \rho_{d}}{d \chi} .
$$

The first condition and the conservation equations give

$$
\frac{d \phi}{d \chi}=\frac{6}{q}\left(\Omega_{V}-\Omega_{k}\right)
$$

Expressing $d \phi / d \chi$ through $\Omega_{k}=(d \phi / d \chi)^{2} / 6 \lambda^{2}$, and inserting it in the second condition (3.12), we obtain, respectively,

$$
\lambda q=\sqrt{\frac{6}{\Omega_{k}}}\left(\Omega_{V}-\Omega_{k}\right), \quad q=2 \frac{\Omega_{V}-\Omega_{k}}{1+\Omega_{k}-\Omega_{V}},
$$

where in the latter the asymptotic form of the potential (3.2) has been used. The last two equations can be solved for $\Omega_{k}$ and $\Omega_{V}$,

$$
\Omega_{k}=\frac{6}{\lambda^{2}(2+q)^{2}}, \quad \Omega_{V}=\Omega_{k}+\frac{q}{q+2}
$$

giving easily

$$
\Omega_{\phi}=\frac{12+q(q+2) \lambda^{2}}{(q+2)^{2} \lambda^{2}}, \quad w_{\phi}=-\frac{q(q+2) \lambda^{2}}{12+q(q+2) \lambda^{2}},
$$

where the last equation for $w_{\phi}=\left(\Omega_{k}-\Omega_{V}\right) /\left(\Omega_{k}+\Omega_{V}\right)$ provides the dilaton's equation of state.

The above asymptotic solution, first obained in [32], and recently studied in $[33,34]$, generalizes the results discussed in Ref. [27] to the interacting dark matter case, and is very similar to the results obtained by including suitable nonminimal couplings in a Brans-Dicke context [28], or by including an effective bulk viscosity in the dark matter stress

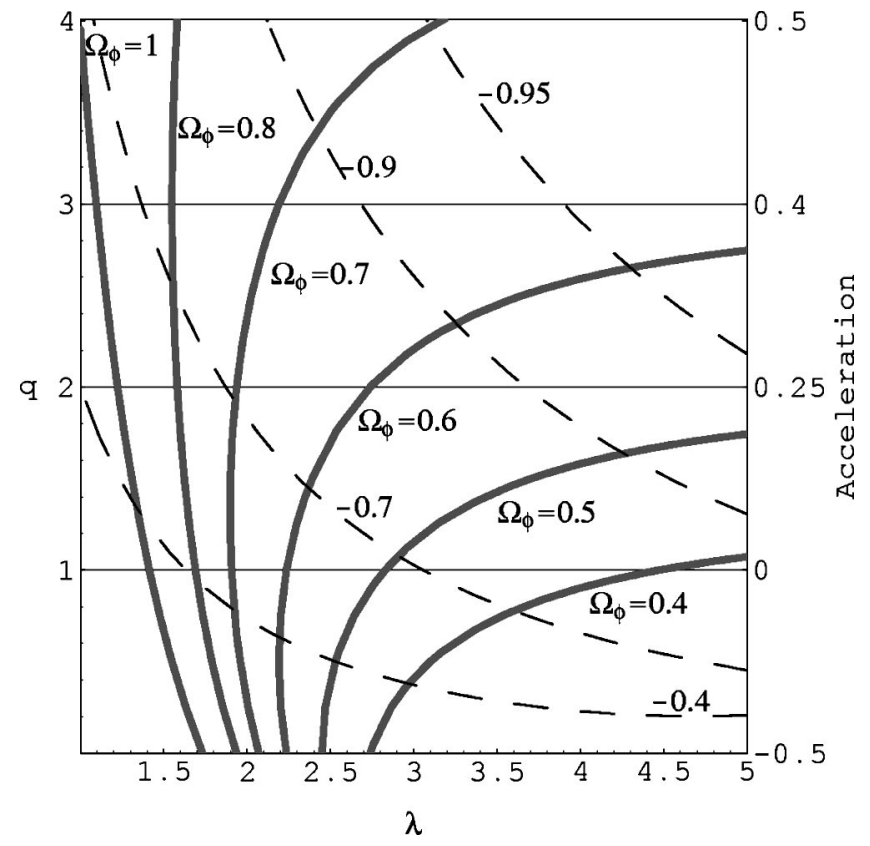

FIG. 1. The asymptotic configurations in the plane $\{\lambda, q\}$. The full bold curves correspond to asymptotic solutions with fixed ratios $\rho_{\phi} / \rho_{d}$ and with the following values of $\Omega_{\phi}: 1,0.8,0.7,0.6,0.5,0.4$. On the right vertical axis we have reported the corresponding $q$-dependent acceleration parameter, $\ddot{a} a / \dot{a}^{2}$. The thin dashed curves correspond to fixed asymptotic values of the dilatonic equation of state $w_{\phi}=p_{\phi} / \rho_{\phi}$, respectively $-0.4,-0.7,-0.9$ and -0.95 .

tensor [10,35]. Our Eq. (3.9) corresponds indeed, formally, to a dissipative pressure $\Pi=-q \rho_{m}(\dot{\phi} / 6 H)$ (in the notation of [35]). See also $[33,34]$ for a discussion of the parameter values compatible with such an asymptotic solution.

Once $\Omega_{k}$ and $\Omega_{V}$ are given, one can easily compute all the relevant kinematic properties of the asymptotic solution as a function of only two parameters, $q$ and $\lambda=c_{1} / c_{2}$, which are in principle calculable for a given string theory model. The asymptotic value of the acceleration, in particular, is fixed by Eq. (3.10) as

$$
\frac{\ddot{a}}{a H^{2}}=1+\frac{\dot{H}}{H^{2}}=\frac{q-1}{q+2} .
$$

One can also easily obtain, through a simple integration, the asymptotic evolution of the Hubble factor and of the dominant energy density,

$$
H \sim a^{-3 /(2+q)}, \quad \rho \sim a^{-6 /(2+q)} .
$$

In order to illustrate the range of parameters possibly compatible with present phenomenology, we have plotted in the $\{\lambda, q\}$ plane various curves at $\Omega_{\phi}=\Omega_{k}+\Omega_{V}=$ const, and $w_{\phi}=\left(\Omega_{k}-\Omega_{V}\right) /\left(\Omega_{k}+\Omega_{V}\right)=$ const (Fig. 1). Note that the case discussed in Ref. [27] corresponds to staying on the $\lambda$ axis. In that case, the critical value of $\lambda$ below which $\Omega_{d} / \Omega_{\phi} \rightarrow 0$ is $\sqrt{3}$. The addition of $q$ makes parameter space two-dimensional, with the point $\lambda=\sqrt{3}$ replaced by the leftmost curve $\Omega_{\phi}=1$. Beyond that curve, i.e. for $\lambda^{2}<6 /(2$ 
$+q$ ) (as well as for all values of $\lambda$ if $q<-2$ ), the ratio $\Omega_{d} / \Omega_{\phi}$ goes to zero. However, while in the case of [27] acceleration and a finite ratio $\Omega_{d} / \Omega_{\phi}$ are incompatible, this is perfectly possible in a large region of the $\{\lambda, q\}$ plane.

In fact, it is possible to determine the region of our parameter space that survives the various observational constraints (type 1a supernovae, CMB anisotropies, large-scale structure ...). The present values of $\Omega_{\phi}$ and $w_{\phi}$ have to lie in the range $[36,37] 0.6 \lesssim \Omega_{\phi} \leqq 0.7$, and $-1 \leqslant w_{\phi} \lesssim-0.4$, but the two allowed intervals are not uncorrelated. Assuming that we are already in the asymptotic regime, the allowed region lies roughly between the two curves $\Omega_{\phi}=0.6$ and $\Omega_{\phi}=0.7$ and above $q=2$. Other phenomenological (but somewhat more model-dependent) constraints on $q$ and $\lambda$ can be obtained from the recent measurements of the position of the third anisotropy peak in the CMB distribution [38], which constrains the value of $\Omega_{\phi}$ today and at last scattering, as well as the time-averaged equation of state $\left\langle w_{\phi}\right\rangle$ [39]. In the final part of this paper we shall present a model of dark matter that seems to be compatible with all the abovementioned constraints.

\section{FOCUSING AND DRAGGING WITH $V=0$ : AN ANALYTIC STUDY OF EARLY-TIME EVOLUTION}

Having discussed, in the previous section, the late-time accelerated expansion caused by the interplay of the dilaton potential and the dark-matter dilatonic charge, it looks appropriate to illustrate the earlier evolution, i.e. before the dilaton potential starts entering the game. In this section we shall provide a semi-quantitative, analytic analysis of this behavior as it follows from the string cosmology equations (2.21)(2.24), by imposing on the non-perturbative normalization (3.3) the constraint $V_{0}^{1 / 4} \ll H_{\text {eq }}$, where $H_{\text {eq }}$ is the curvature scale at the epoch of matter-radiation equality. In such a way the dilaton potential may eventually become important only at late times, in the matter-dominated era. We will show that this early evolution can be roughly divided in three epochs, providing, altogether, an intermediate attractor that nicely connects to the accelerated behavior described in Sec. III.

Let us start by considering an initial, post-big bang and post-inflationary regime of expansion driven by the standard radiation fluid, with negligible dilatonic charge, $\sigma_{r}=0$. Possible non-relativistic matter, if present, is highly subdominant with respect to the other components $\left(\rho_{m} \ll \rho_{\phi}, \rho_{r}\right)$ and, consequently, the dilatonic terms in Eq. (2.24) can be neglected. The conservation equations can be easily integrated to give

$$
\rho_{r}=\rho_{r i} e^{-4 \chi}, \quad \rho_{\phi}=\rho_{\phi i} e^{-6 \chi} .
$$

Therefore, the dilaton (kinetic) energy density, even if initially of the same order as $\rho_{r}$, is rapidly diluted like $a^{-6}$. The dilaton itself, starting from a value $\phi_{i} \sim \mathcal{O}(1)$ typical of the moderately-strong coupling post-big bang epoch, tends to settle down to a constant value (as already noticed in [40]), that can be easily estimated as follows

$$
\rho_{k}=\frac{k^{2}}{2} H^{2}\left(\frac{d \phi}{d \chi}\right)^{2}=\frac{k^{2}}{12}\left(\frac{d \phi}{d \chi}\right)^{2}\left(\rho_{r}+\rho_{\phi}\right)=\rho_{\phi} .
$$

For $\rho_{r i}=\rho_{\phi i}$ we get

$$
\frac{d \phi}{d \chi}=\sqrt{\frac{12}{k}}\left(1+e^{2 \chi}\right)^{-1 / 2},
$$

which, for $k=$ const, leads to a solution with asymptotic value $\phi=\phi_{1}$, related to the initial value $\phi_{i}=\phi(0)$ by the constant shift

$$
\Delta \phi=\phi_{1}-\phi_{i}=\sqrt{\frac{12}{k}} \ln (1+\sqrt{2}) \simeq \frac{3}{k} \simeq \frac{3}{\sqrt{2}} \frac{c_{1}}{c_{2}},
$$

independently of $\phi_{i}$ and of the initial $\chi$ (the last equality holds for $\phi_{1}$ large enough to justify the asymptotic relation $k=\sqrt{2} / \lambda)$.

Such an initial regime is effective until the dilaton kinetic energy becomes of the same order as $\rho_{m}$. At that point, some oscillations are triggered by the interference term of Eq. (2.24), but the dilaton energy density keeps decreasing, on the average, until it enters a "focusing" regime, during which it is diluted at a much slower rate (like $a^{-2}$ ), so as to converge, at equality, towards the larger values of $\rho_{m}$ and $\rho_{r}$. Eventually, when dark non-relativistic matter becomes the dominant source $\left(\rho_{d} \gtrsim \rho_{r}\right)$, the dilaton energy density tends to follow the dark matter evolution, as if it were "dragged" by it.

Before turning to a quantitative analysis of these two regimes we note that the time evolution of $\rho_{\phi}$, in the "tracking" quintessence, is determined by the slope of the potential. In the present context, instead, the focusing and dragging effects are not due to the potential, but they are controlled by the non-minimal coupling induced by $\left(\psi^{\prime}\right.$ $+q$ ) (thus implementing an attractor mechanism already proposed for a class of non-minimal scalar-tensor models of quintessence [41]). Thanks to the focusing effect, which seems to be typical of the string effective action (even if similar, in a sense, to the "self-adjusting" solutions of general relativity with exponential potential [27]), the dilaton energy density at the matter-radiation equality turns out to be fixed independently from its initial value, and only slightly dependent on the initial value of the dilaton, $\phi_{i}$. For large enough values of $q$, however, even the dependence upon $\phi_{i}$ tends to disappear, because the value of the dilaton itself gets focused, as will be discussed in the next section.

For a quantitative analytical study of the "focusing" and "dragging" regimes, we start from Eqs. (2.21)-(2.24). Lumping together baryonic and dark matter, neglecting $V$, and assuming, according to Eq. (3.7), $\sigma=\sigma_{m}=q(\phi) \rho_{m}$, those equations can be easily recast in the form:

$$
\begin{array}{r}
\rho_{r}^{-1} \frac{d \rho_{r}}{d \chi}+4=0, \\
\rho_{m}^{-1} \frac{d \rho_{m}}{d \chi}+\left[3 \mp \sqrt{3} \epsilon\left(\rho_{\phi} / \rho\right)^{1 / 2}\right]=0, \\
\frac{d \rho_{\phi}}{d \chi}+6 \rho_{\phi} \pm \sqrt{3} \epsilon \rho_{m}\left(\rho_{\phi} / \rho\right)^{1 / 2}=0,
\end{array}
$$


where we have introduced the important parameter

$$
\epsilon(\phi) \equiv \frac{\psi^{\prime}(\phi)+q(\phi)}{k(\phi)},
$$

and the sign ambiguity comes from solving Eq. (4.2) for $d \phi / d \chi$ in terms of $\rho_{\phi}$. The focusing solution is then characterized by the relation

$$
\rho_{\phi}=\frac{n^{2}(\phi) \rho_{m}^{2}}{\rho}
$$

i.e. $\Omega_{\phi}=n^{2}(\phi) \Omega_{m}^{2}$, which holds under the assumption that both $\epsilon$ and $n$ are slowly varying. Indeed, we can establish the connection between these two quantities by inserting the ansatz (4.9) into (4.7). This gives:

$$
-6 \mp \sqrt{3} \frac{\epsilon}{n}=2 n^{-1} \frac{d n}{d \chi}+2 \rho_{m}^{-1} \frac{d \rho_{m}}{d \chi}-\rho^{-1} \frac{d \rho}{d \chi},
$$

where on the right-hand side the logarithmic derivative of Eq. (4.9) has been taken. By using Eq. (4.6) one finally has

$$
\rho^{-1} \frac{d \rho}{d \chi} \mp \sqrt{3} \epsilon\left[n^{-1}+2 \Omega_{m} n\right]=2 n^{-1} \frac{d n}{d \chi} \simeq 0 .
$$

We can now discuss a few cases of interest. During the radiation-dominated phase, and after the kinetic energy of the dilaton is quickly red-shifted away, we can neglect the term with $\Omega_{m}$ in Eq. (4.11), we set $d \rho / d \chi=-4 \rho$, and obtain:

$$
n \simeq \frac{\sqrt{3} \epsilon}{4}, \quad \frac{d \phi}{d \chi} \simeq-\frac{3 \rho_{m} \epsilon}{2 k \rho}, \quad \rho_{\phi} \simeq \frac{3 \rho_{m}^{2} \epsilon^{2}}{16 \rho} .
$$

We refer to this behavior as "focusing" since it implies that $\rho_{m}$ lies, modulo a factor $(16 / 3) \epsilon^{-2}$, at the geometric mean between $\rho \sim \rho_{r}$ and $\rho_{\phi}$. Hence, as we approach radiationmatter equality, $\rho_{\phi}$ is effectively focused towards the same common value of the other two components [see Eq. (4.15) below]. Note that, for a positive $\epsilon$, this happens thanks to a negative $d \phi / d \chi$.

In the matter-dominated regime it is no longer safe to neglect the term in $\Omega_{m}$ in Eq. (4.11), unless $\epsilon \ll 1$. In that case, the solution is

$$
n \simeq \sqrt{3} \epsilon\left(-\rho^{-1} \frac{d \rho}{d \chi}\right)^{-1}, \quad \Omega_{\phi} \simeq 3 \epsilon^{2} \Omega_{m}^{2}\left(-\rho^{-1} \frac{d \rho}{d \chi}\right)^{-2} .
$$

During matter domination, using $d \rho / d \chi=-3 \rho$, one gets

$$
n \simeq \frac{\epsilon}{\sqrt{3}}, \quad \frac{d \phi}{d \chi} \simeq-\frac{2 \epsilon}{k}, \quad \rho_{\phi} \simeq \frac{\rho_{m} \epsilon^{2}}{3} .
$$

In other words, the focusing regime has been turned into a dragging one: the dilaton energy is dragged along by the (dark) matter energy and keeps a (small) constant ratio to it.
Incidentally, at the epoch of exact matter-radiation equality, using $d \rho / d \chi=-3.5 \rho$, we easily get (still at small $\epsilon$ ):

$$
\frac{\rho_{\phi}}{\rho_{e q}} \simeq \frac{3 \epsilon^{2}}{49}
$$

which is always smaller than $6 \%$ for $\epsilon<1$.

In order to understand what happens at larger values of $\epsilon$ it is useful to find the reason why, for small $\epsilon, \rho_{\phi} / \rho_{m}$ stays constant. This comes about because the corrections to the $a^{-3}$ and $a^{-6}$ laws for $\rho_{m}$ and $\rho_{\phi}$, due to the non-vanishing $\epsilon$, push the two towards each other. It is easy to check that, precisely if $\rho_{\phi} /\left(\rho_{m}+\rho_{\phi}\right)=\epsilon^{2} / 3$, both energies scale like $a^{-\left(3+\epsilon^{2}\right)}$. We note, incidentally, that the above ratio of energies nicely fits with the value given in Eq. (4.14) when $\epsilon$ $\ll 1$. If $\epsilon<1$, the decrease of $\rho_{\phi}$ is still slower than the $a^{-4}$ of $\rho_{r}$, which justifies neglecting the latter. However, if $\epsilon$ $>1$, this is no longer the case and we have a third kind of behavior, which can be called "total dragging." In that case, as shown by a simple analysis, all three components of $\rho$ scale like radiation, with the following sharing of the "energy budget" (remember that we are always at $\Omega=1$ ):

$$
\Omega_{\phi}=\frac{\Omega_{m}}{2}=\frac{1}{3 \epsilon^{2}}, \quad \Omega_{r}=\frac{\epsilon^{2}-1}{\epsilon^{2}} .
$$

In the next section we will see how numerical integration confirms in full detail the analytic behavior we have discussed. We end this section by discussing some constraints on our parameters.

As already mentioned, we assume the ordinary components of matter (radiation and baryons) to have a nearly metric coupling to $\widetilde{g}_{\mu \nu}$ [see the discussion after Eq. (3.4)]. To be more specific, let us define the ratios between dilatonic charges and energy densities in a way similar to that used for cold dark matter in Eq. (3.7), i.e.

$$
q_{r}(\phi) \equiv \sigma_{r} / \rho_{r}, \quad q_{b}(\phi) \equiv \sigma_{b} / \rho_{b} .
$$

Since it is precisely the ratio $\left(\psi^{\prime}+q_{r, b}\right) / k$, which controls both the effective coupling of the dilaton to ordinary macroscopic matter, as well as a possible time-dependence of the fundamental constants $[42,43]$, we shall assume that both $q_{b}$ and $q_{r}$ are at most of order $\psi^{\prime}$, in agreement with the discussion after Eq. (3.5). We then find that there are neither appreciable violations of the equivalence principle in the context of macroscopic gravitational interactions, nor significant contributions to the time-variation of the fundamental constants, both effects being controlled by $\psi^{\prime} / k$ for $q_{r, b}$ $\rightarrow 0$. In the strong coupling regime we have $\psi^{\prime} / k$ $\sim e^{-\phi} /\left(c_{1} c_{2}\right)$. For a non-negative $\phi_{i}$, and $c_{1}^{2}, c_{2}^{2}$ of order $10^{2}$, there is no appreciable deviation from the standard cosmological scenario down to the epoch of matter-radiation equality, so that one easily satisfies the early-Universe constraints on dark energy, as reported for instance in [44].

The dilaton charge of dark matter is not restricted by the experimental tests of long-range gravitational interactions: this is the reason why we can play with it in order to produce an acceleration. Still, from the above discussion on the early 
phases of the Universe, it is clear that high values of the dark-matter parameter $\epsilon$ may result in dangerously high values for $\Omega_{\phi}$, and thus in radical deviations from the standard cosmological scenario. Until radiation-matter equality the situation is relatively harmless: we can easily estimate the dilaton energy density at the equality and at the nucleosynthesis scale, $H_{N} \sim 10{ }^{10} H_{\text {eq }}$, using the fact that the dilaton, during the focusing regime, is not significantly shifted away from the value $\phi_{i}+\Delta \phi$, fixed by Eq. (4.4). Because of the focusing behavior we find $\Omega_{\phi}$ (nucl) $\sim 10^{-10} \Omega_{\phi}(\mathrm{eq})$, and therefore the most stringent bound comes at equality, where, thanks to Eq. (4.15), it is comfortably satisfied for $\epsilon<1$.

During the dragging phase, however, we must certainly impose $\epsilon<1$; otherwise, the phenomenon of "total dragging" takes place. This would represent a dramatic deviation from the standard cosmological scenario, since all the components $\rho_{\phi}, \rho_{r}, \rho_{d}$ (except baryonic matter) would redshift in the same way $\left(a^{-4}\right)$ from equality until the potential starts to be felt. Even if $\epsilon<1$, but not sufficiently small, the unusual scaling $\rho_{m} \propto a^{-3-\epsilon^{2}}$ tends to change the global temporal picture between now and the epoch of matter-radiation equality and, from Eqs. (4.14), values of $\Omega_{\phi} \sim \epsilon^{2} / 3$ (while in agreement with possible constraints at last scattering [44]) can be dangerously high. In our context, a bound $\Omega_{\phi}($ drag $)<0.1$, i.e. $\epsilon($ drag $)<0.3$, appears to be necessary in order to agree with the observed CMB spectrum and with the standard scenario of structure formation.

On the other hand, due to the smallness of $\psi^{\prime} \sim e^{-\phi} / c_{1}^{2}$ in the dragging regime, an upper bound on $\epsilon$ effectively turns into a bound on the value of $q / k$, i.e. on the dilatonic charge of the dark matter component. The above constraints thus translate into a bound for the combination $\lambda q$ :

$$
\lambda q\left(\phi_{\mathrm{drag}}\right)<0.8,
$$

where we used the already mentioned asymptotic relation $\lambda$ $=\sqrt{2} / \mathrm{k}$. It is clear that a constant $q$ cannot satisfy the above bound and, at the same time, provide the present acceleration of the Universe by means of the mechanism described in Sec. III (see also Fig. 1), that requires $q \lambda \geqslant 4$.

A time- (or, better, $\phi$-) dependent $q$, however, is allowed. For this reason we have to consider cold dark matter models like the one of Eq. (3.4), whose dilatonic charge (3.7) switches on at large enough values of the dilaton. The transition to large values of $\phi$ is rapidly activated as the potential comes into play, $\rho_{V} \sim \rho_{\phi}$. At that point, the dilaton energy density stops decreasing and freezes at a constant value, necessarily crossing, at some later moment, the matter energy density, $\rho_{\phi} \sim \rho_{d}$. From then on, the dilaton starts rolling towards $+\infty$, triggering the effect of the dilatonic charge, which rapidly freezes the ratio $\rho_{\phi} / \rho_{m}$ and (for suitable values of $q$ ) leads to the accelerated asymptotic regime described by Eqs. (3.16), (3.17). Explicit numerical examples of such a behavior will be discussed in Sec. V.

For a realistic picture, in which the positive acceleration regime starts around the present epoch (and not much earlier) and the standard scenario of structure formation is implemented successfully, we have to require that the contribution of the dilatonic charge (as well as the effect of the dilaton potential) come into play only at a late enough epoch. The importance of this constraint was already discussed in the context of other scalar-tensor models of quintessence [28] where, for instance, the non-minimal coupling of the scalar field to the trace of the dark matter stress tensor was assumed to be $\phi$-dependent, to interpolate between a small and a large mixing regime.

\section{NUMERICAL EXAMPLES}

Finally, after the analytic discussion of the previous section, it seems appropriate to illustrate the "runaway" dilaton scenario with some numerical example, both in order to confirm the validity of some approximations made in deriving the analytic results, and in order to see how the various regimes we discussed can be put together. To this aim, we shall numerically integrate Eqs. (2.19)-(2.23), using Eq. (2.20) as a constraint on the set of initial data, and assuming an explicit model for the dilatonic charges and the dilaton potential. Also, following the "induced-gravity" ideas [21], we shall specialize the loop form-factors according to Eq. (3.1), using the "minimal" choice

$$
e^{-\psi(\phi)}=e^{-\phi}+c_{1}^{2}, \quad Z(\phi)=e^{-\phi}-c_{2}^{2} .
$$

First of all, for a clear illustration of the "focusing" and "dragging" regimes, let us put $V=0, \sigma_{r}=0=\sigma_{b}$, and $\sigma_{d}$ $=q \rho_{d}$, with $q=$ const. By choosing, in particular, $c_{1}^{2}=100$, $c_{2}^{2}=30$, we have integrated Eqs. (2.19) $-(2.23)$ for three different values of the charge, $q=0, q=0.01$, and $q=0.1$, starting from the initial scale $H_{i}=10^{40} H_{\text {eq }}$,

$$
\left(\frac{a_{i}}{a_{\mathrm{eq}}}\right)=\left(\frac{H_{\mathrm{eq}}}{H_{i}}\right)^{1 / 2}=\left(\frac{\rho_{m i}}{\rho_{r i}}\right)=10^{-20},
$$

and using $\rho_{\phi i}=\rho_{r i}, \phi_{i}=-2$ as initial conditions. It should be noted that such initial conditions are generic, in the sense that different initial values of $\rho_{\phi}$ and $\phi$ may change the fixed value reached by $\phi$ during the focusing phase, but do not affect in a significant way the subsequent evolution, as will be discussed at the end of this section.

The results of this first numerical integration are illustrated in Fig. 2. The left panel clearly displays the initial regime of fast dilaton dilution $\left(\rho_{\phi} \sim a^{-6}\right)$, the subsequent focusing regime $\left[\rho_{\phi} \sim a^{-2}\right.$, see Eq. (4.12)] triggered (after some oscillations) soon after $\rho_{\phi}$ falls below $\rho_{m}$, and the final dragging regime $\left[\rho_{\phi} \sim \rho_{m}\right.$, see Eq. (4.14)] in the epoch of matter domination (the epoch of matter-radiation equality corresponds to $\chi \simeq 46$ ). Note that the constant values of $q$ have been chosen small enough to avoid the phenomenon of "total dragging," see Sec. IV. Note also that, in this example, $\rho_{m}$ always coincides with $\rho_{d}$. In the right panel the evolution of $\Omega_{\phi}$, obtained through the numerical integration, is compared with the analytic estimates (4.12), (4.13), (4.14), for the three different values of $q$. In all cases, $\Omega_{\phi}$ grows like $a^{2}$ during the focusing regime (in the radiation era), while the final stabilization $\Omega_{\phi}=$ const, after the epoch of matter- 

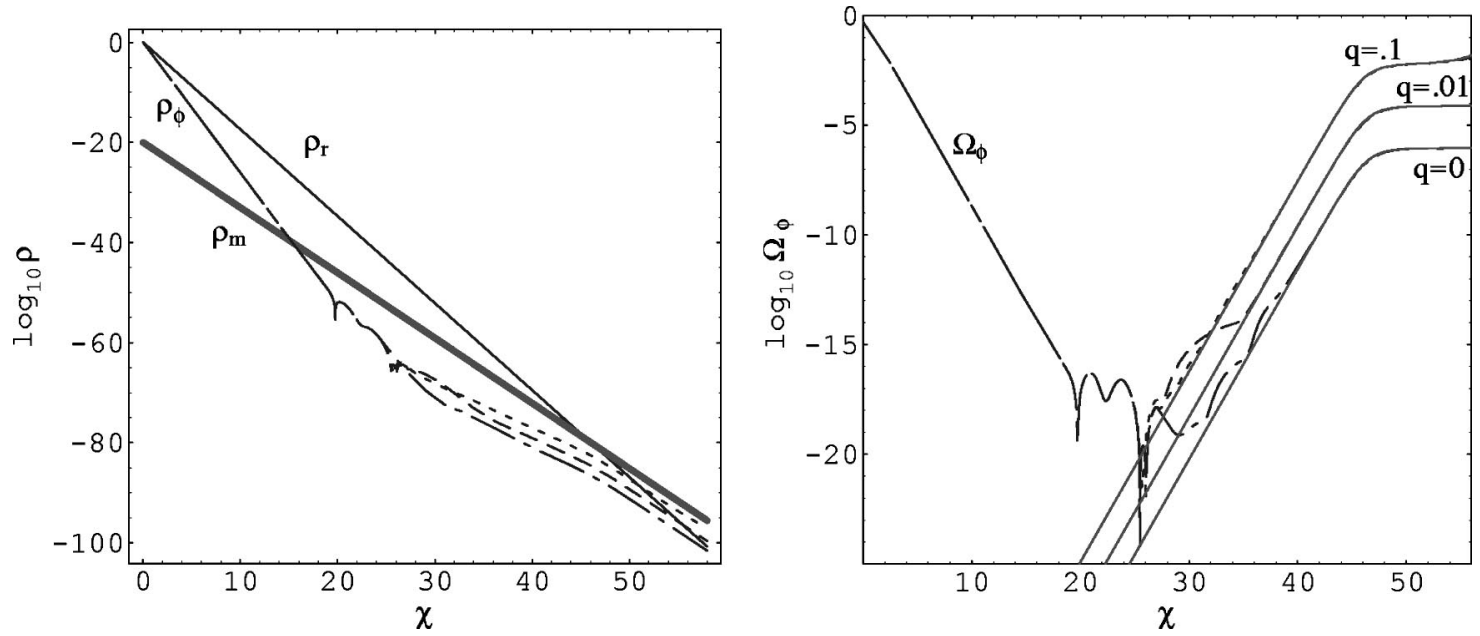

FIG. 2. Time evolution of $\rho_{\phi}$ for $q=0$ (dash-dotted curve), $q=0.01$ (dashed curve) and $q=0.1$ (dotted curve). The initial scale is $a_{i}$ $=10^{-20} a_{\mathrm{eq}}$, and the epoch of matter-radiation equality corresponds to $\chi \simeq 46$. Left panel: the dilaton energy density is compared with the radiation (thin solid curve) and matter (bold solid curve) energy density. Right panel: the dilaton energy density (in critical units) is compared with the analytical estimates (4.12), (4.13), (4.14) for the focusing and dragging phases.

radiation equality ( $\chi \gtrsim 46)$, clearly illustrates the effect of the dragging phase during which $\rho_{\phi}$ and $\rho_{m}$ evolve in time with the same behavior.

For a realistic model of quintessence, however, a constant dilatonic charge cannot drive the Universe towards an asymptotic accelerated regime and, simultaneously, satisfy all the required phenomenological constraints during the earlier epochs (as discussed in the previous sections). By keeping $\sigma_{b}, \sigma_{r} \simeq 0$ at large coupling [see Eq. (3.5) and the discussion thereafter], we shall thus consider the explicit model of scalar dark matter (3.4), with the following simple loop form-factors

$$
e^{-\zeta(\phi)}=1+e^{q_{0} \phi} / c^{2}, \quad e^{\eta(\phi)}=\mathrm{const}
$$

(note that, by a field redefinition, one of the two loop factors can always be taken to be trivial: what really matters is the ratio $e^{\zeta} / e^{\eta}$ ). Using Eq. (3.7) we immediately get

$$
q(\phi)=\frac{\sigma_{d}}{\rho_{d}}=q_{0} \frac{e^{q_{0} \phi}}{c^{2}+e^{q_{0} \phi}},
$$

which is exponentially suppressed in the perturbative regime, and approaches $q=q_{0}$ at large coupling (for $q_{0}>1$ it is thus compatible with an asymptotically accelerated cosmological configuration, see Fig. 1). For our numerical example we shall choose $q_{0}=2.5$ and $c^{2}=150$, but the behavior of the solution is rather stable, at late times, against large variations of the latter parameter (see the discussion at the end of this section).

In addition, we have to specify the form of the dilaton potential. In agreement with its non-perturbative origin, and with the assumtion of exponential suppression at strong coupling (see Sec. IV), the simplest choice is a difference of terms of the type $e^{-\beta / \alpha(\phi)}$. We shall thus consider the belllike potential (in units $M_{P}^{2}=2$ )

$$
\begin{gathered}
V(\phi)=m_{V}^{2}\left[\exp \left(-e^{-\phi} / \beta_{1}\right)-\exp \left(-e^{-\phi} / \beta_{2}\right)\right], \\
0<\beta_{2}<\beta_{1},
\end{gathered}
$$

which leads, asymptotically, to the large- $\phi$ behavior of Eq. (3.2). The mass scale $m_{V}$, related to the mass $M_{*}$ of Eq. (3.3), will be fixed at $m_{V}=10^{-3} H_{\text {eq }}$, together with $\beta_{1}$ $=10, \beta_{2}=5$, for a realistic scenario that starts accelerating at a phenomenologically acceptable epoch.

With all the parameters fixed, we have numerically integrated the evolution equations (2.19)-(2.23), for our model of charge (5.4) and potential (5.5), using the same initial conditions as in the previous example, but separating the dark and baryonic components inside $\rho_{m}$. In particular, we have set, initially, $\rho_{d i}=10^{-20} \rho_{r i}, \rho_{b i}=7 \times 10^{-21} \rho_{r i}$.

The resulting late-time evolution of the various energy densities is shown in the left panel of Fig. 3. Dark matter and baryonic energy densities evolve in the same way, until the potential comes into play, starting at a scale around $\chi \simeq 49$. The potential first tends to stabilize $\rho_{\phi}$ to a constant but then (thanks to the contribution of $q$ ) the system eventually evolves towards a final regime in which $\rho_{\phi}$ and $\rho_{d}$ are closely tied up, and their asymptotic evolution departs from the trajectory of the standard, decelerated scenario [in particular, they both scale, asymptotically as $a^{-6 /\left(2+q_{0}\right)}$, see Eq. (3.18)]. It is amusing to conjecture that the different timedependence of $\rho_{b}$ and $r_{d}$ could be responsible for the present small ratio $\rho_{b} / \rho_{d}$.

In the right panel we have plotted the time evolution of the dilatonic charge $q$, of the energy density $\Omega_{\phi}$, of the equation of state $w_{\phi}$, and of the acceleration parameter $\ddot{a} / a H^{2}$. When the potential energy becomes important, all the above quantities rapidly approach their asymptotic values given in Eqs. (3.16),(3.17). Note that, with our choice of parameters, we have $q_{0}=2.5$ and $\lambda=c_{1} / c_{2}=\sqrt{10 / 3}$, corresponding to an asymptotic value $\Omega_{\phi} \simeq 0.733$, slightly exceeding the best fit value suggested by present observations 

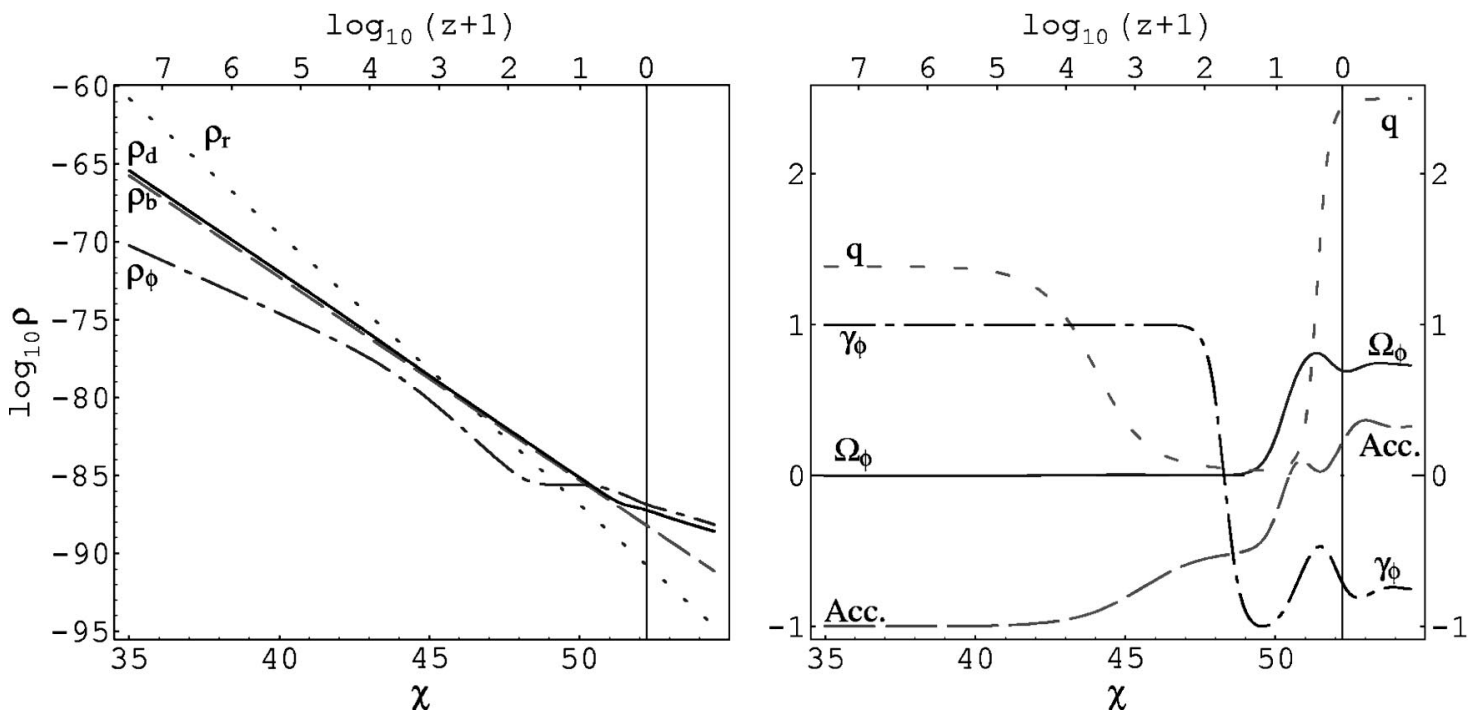

FIG. 3. Left panel: Late-time evolution of the dark matter (solid curve), barionic matter (dashed curve), radiation (dotted curve) and the dilaton (dash-dotted curve) energy densities, for the string cosmology model specified by Eqs. (5.4),(5.5). The upper horizontal axis gives the $\log _{10}$ of the redshift parameter. Right panel: for the same model, the late-time evolution of $q$ (fine-dashed curve), $w_{\phi}\left(\right.$ dash-dotted curve), $\Omega_{\phi}$ (solid curve) and of the acceleration parameter $\ddot{a} a / \dot{a}^{2}$ (dashed curve).

$[36,37]$. It is important to stress, however, that the asymptotic attractor may be preceded by a (short) oscillating regime, which, as illustrated in the right panel of Fig. 3, can easily allow for values of the cosmological parameters different from the asymptotic ones to be compatible with present observations. Note also that, when switching from the focusing to the dragging phases, the dilaton starts to move back towards decreasing values of $q$, as will be illustrated also by a subsequent numerical integration. This may slow down the evolution of $\rho_{\phi}$ with respect to $\rho_{m}$ during the dragging, as

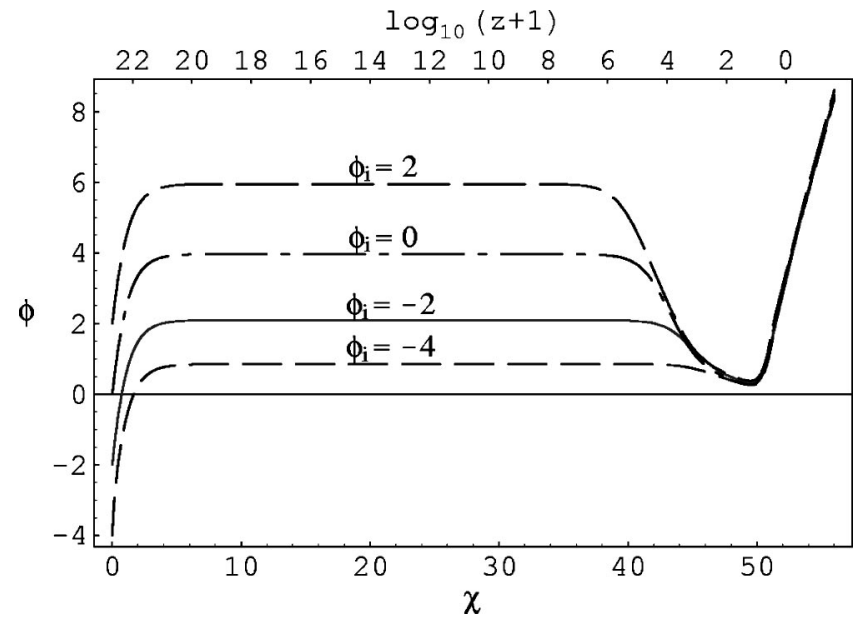

FIG. 4. Time evolution of the dilaton field, for different initial conditions $\phi_{i}=-4,-2,0,2$. All the other parameters are the same as in the example of Fig. 3. After the plateau associated with the focusing regime, and for a strong enough dilatonic charge, the solutions tend to converge to a common value of $\phi$. The subsequent running to $+\infty$, driven by the potential, is thus completely independent of the initial value. shown for instance in the left panel of Fig. 3. Because of this effect, however, the dilaton can easily satisfy, during the dragging phase, the phenomenological bounds discussed in the previous sections. This does not require fine tuning, the validity of the bounds being guaranteed for a large basin of initial conditions by a convergent behavior of the solutions during dragging.

During the focusing phase, in fact, the dilaton is practically frozen, as can be argued from Eq. (4.12), and its effective constant value, as determined by Eq. (4.4), depends on $\phi_{i}$. However, if such a value is high enough, the presence of the dilatonic charge may become important, and may contribute to the focalization towards the epoch of matterradiation equality, as already anticipated. This is illustrated in Fig. 4, which shows the time evolution of the dilaton obtained by numerically integrating the same model as in Fig. 3 , for different initial values $\phi_{i}=-4,-2,0,2$. Although we start with different dilaton values at the plateau associated with the focusing regime, all the solutions tend to converge as we enter the dragging regime, so as to make the subsequent (potential-dominated) evolution insensitive to the initial value of the dilaton. ${ }^{2}$

This new focusing effect, which is very different from the one of the energy densities during the radiation-dominated phase, can also be understood analytically by writing the solution of Eq. (4.14) as

$$
\chi^{-\chi_{\mathrm{eq}}}=-\int_{\phi_{\mathrm{eq}}}^{\phi} \frac{k(\bar{\phi})}{2 \epsilon(\bar{\phi})} d \bar{\phi}
$$

\footnotetext{
${ }^{2}$ The preceding evolution, of course, is not sensitive either, since during focusing the order of magnitude of $\Omega_{\phi}$ is given by $\Omega_{m}^{2}$ as in Eq. (4.12).
} 


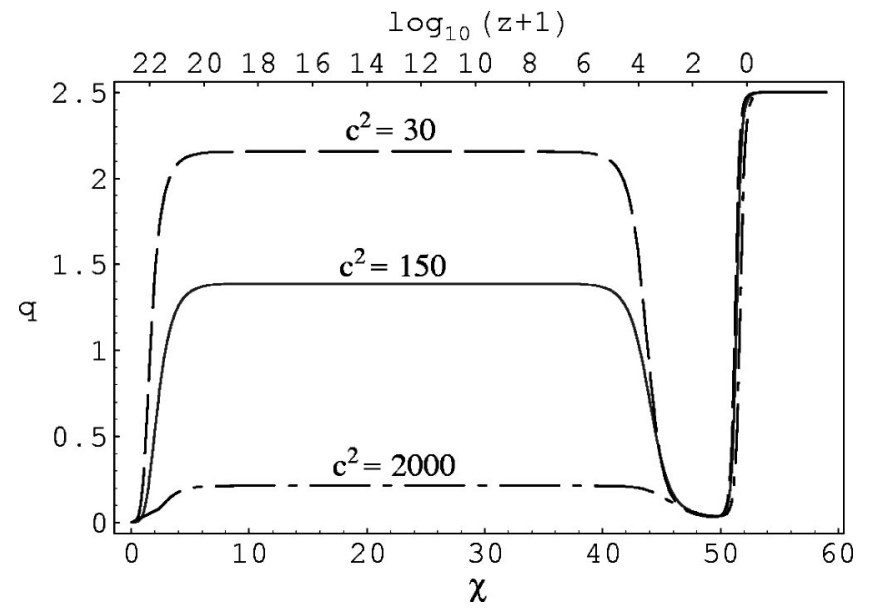

FIG. 5. Time evolution of $q(\phi)$, from Eq. (5.4), for three different values of the parameter $c$. All the other parameters are the same as in the example of Fig. 3. During the dragging phase the value of $q$ converges to the regime $q \ll 1$.

Since $k$ is almost constant, a variation $\delta \phi_{\text {eq }}$ on the initial value of $\phi$ changes the solution $\phi(\chi)$ by an amount $\delta \phi(\chi)=\left[\epsilon(\phi) / \epsilon\left(\phi_{\text {eq }}\right)\right] \delta \phi_{\text {eq }}$, which rapidly decreases [with $q(\phi)$ ] during the dragging phase. This is why the solution has become independent of the initial value of $\phi$ by the time the potential becomes an important component.

For the same reason, the model is only weakly affected by variations of the parameter $c$ in Eq. (5.4), which roughly gives the transition scale between small and large dilatonic charges: $\phi_{s}=\left(2 / q_{0}\right) \log c$. Indeed, because of the above mechanism the dilaton is pushed back during the dragging phase, with a velocity as high as needed to reach, in any case, the safe zone $q \ll 1$. This effect is illustrated in Fig. 5, where we have plotted the time evolution of $q(\phi)$, for the same model as Fig. 3, and for three different values of $c$.

It should be noted, in conclusion, that the above class of models depends in a crucial way on three important parameters: $m_{V}, q_{0}$ and the ratio $\lambda=c_{1} / c_{2}$. The first one controls the transition time between the epoch of standard cosmological evolution and the final accelerated regime (as can be easily checked, for instance, by repeating the numerical integration of Fig. 3 with different values of $m_{V}$ ). The other two parameters control the asymptotic properties of the model (acceleration, equation of state, ... ), as discussed in Sec. III. Future precision data, both from supernovae observations and from measurements of the CMB anisotropy, could give us a good determination of these parameters, thus providing important indirect information on the parameters of the string effective action in the strong coupling regime.

\section{CONCLUSION}

Let us conclude by summarizing the main points of our work. We have argued that a runaway dilaton can provide an interesting model of quintessence under a well-defined set of assumptions that we list hereafter:

The limit of superstring theory, as its bare four- dimensional effective coupling goes to infinity (so called induced gravity/gauge or compositeness limit), should exist and make sense phenomenologically, i.e. should yield reasonable values for the unified gauge coupling at the GUT scale and for the ratio $M_{P} / M_{G U T}$, thanks to the large number of degrees of freedom at $M_{G U T}$.

In the visible-matter sector, the couplings to the dilaton, either direct or through the trace of the energy-momentum tensor (i.e. via a conformally rescaled metric), should vanish in the $\phi \rightarrow+\infty$ limit.

In the dark matter sector, there should be a surviving coupling to the dilaton (and thus violations of the strong and/or weak equivalence principles) even in the $\phi \rightarrow+\infty$ limit.

The dilaton potential should be non-perturbative, go to zero asymptotically, and have an absolute scale not too far from the present energy density.

Under these circumstances, it is natural for the dilaton energy in critical units, $\Omega_{\phi}$, to be (i) subdominant during radiation domination; (ii) a (small) fraction of the total energy at matter-radiation equality; (iii) a (small) fraction of $\Omega_{m}$ during the earlier epoch of matter domination; (iv) a fraction of dark-matter energy since a redshift $\mathcal{O}(1)$. This very last phase is characterized by an accelerated expansion.

In other words, this framework seems to be naturally consistent with present astrophysical observations and with known cosmological constraints. From a theoretical point of view the model appears to combine nicely previous ideas [15] on dilaton stabilization and decoupling with those recently advocated (e.g. in [28]) so as to have acceleration while keeping the ratio $\Omega$ (dark energy) $/ \Omega$ (dark matter) constant.

It must be stressed, of course, that the analysis presented in this paper is still preliminary, and that various problems are still open. In particular, a precise computation of the CMB anisotropy spectrum, and of the spacing of acoustic peaks expected in this context, as well as a comparison with currently available measurements [4], could provide significant bounds on the parameters of the string cosmology models we have discussed. Such an investigation is postponed to future work. Nevertheless, we believe that the results of this paper are encouraging, as they suggest that the dilaton, which can hardly play the role of the inflaton in the standard inflationary scenario [45], could play instead a successful role as the quintessential field in post-inflationary, late-time cosmology.

\section{ACKNOWLEDGMENTS}

It is a pleasure to thank Luca Amendola, Thibault Damour, Michael Joyce, Alessandro Melchiorri, and J. P. Uzan for useful discussions. G.V. wishes to acknowledge the support of a "Chaire Internationale Blaise Pascal," administered by the "Fondation de L'Ecole Normale Supérieure," during most of this work. F.P. would like to thank Luciano Girardello for his kind support, the "Center Francais pour l'accueil et les échanges internationaux" for financial support, and the "Laboratoire de Physique Théorique, Université Paris Sud, Orsay" for hospitality. 
[1] A.G. Riess et al., Astron. J. 116, 1009 (1998); S. Perlmutter et al., Astrophys. J. 517, 565 (1999).

[2] A.G. Riess et al., astro-ph/0104455.

[3] N.A. Bachall, J.P. Ostriker, S. Perlmutter, and P.J. Steinhardt, Science 284, 1481 (1999).

[4] P. de Bernardis et al., Nature (London) 404, 955 (2000).

[5] S. Weinberg, Rev. Mod. Phys. 61, 1 (1989).

[6] P. Steinhardt, in Critical Problems in Physics, edited by V.L. Fitch and D.R. Marlow (Princeton University Press, Princeton, NJ, 1997).

[7] R.R. Caldwell, R. Dave, and P.J. Steinhardt, Phys. Rev. Lett. 80, 1582 (1998); J.A. Frieman and I. Vaga, Phys. Rev. D 57, 4642 (1998); M.S. Turner and M. White, ibid. 56, 4439 (1997).

[8] P. Binètruy, in Proceedings of the LXXI Les Houches Summer School "The Primordial Universe," edited by P. Binètruy, R. Schaeffer, J. Silk, and F. David (Springer-Verlag, Berlin, 2000), p. 397.

[9] P.J.E. Peebles and A. Vilenkin, Phys. Rev. D 59, 063505 (1999); M. Peloso and F. Rosati, J. High Energy Phys. 12, 026 (1999).

[10] L.P. Chimento, A.S. Jakubi, and D. Pavon, Phys. Rev. D 62, 063508 (2000); W. Zimdahl, D.J. Schwarz, A.B. Balakin, and D. Pavon, ibid. 64, 063501 (2001); S. Sen and A.A. Sen, ibid. 63, 124006 (2001); A.A. Sen and S. Sen, Mod. Phys. Lett. A 16, 1303 (2001).

[11] A. Hebecker and C. Wetterich, Phys. Rev. Lett. 85, 3339 (2000); L. Amendola and D. Tocchini-Valentini, Phys. Rev. D 64, 043509 (2001).

[12] J.P. Uzan, Phys. Rev. D 59, 123510 (1999); T. Chiba, ibid. 60, 083508 (1999); L. Amendola, ibid. 60, 043501 (1999); F. Perrotta, C. Baccigalupi, and S. Matarrese, ibid. 61, 023507 (2000); D.J. Holden and D. Wands, ibid. 61, 043506 (2000); N. Bartolo and M. Pietroni, ibid. 61, 023518 (2000); O. Bertolami and P.J. Martins, ibid. 61, 064007 (2000); R. De Ritis, A.A. Marino, C. Rubano, and P. Scudellaro, ibid. 62, 043506 (2000); R. Bean and J. Magueijo, Phys. Lett. B 517, 177 (2001); S. Sen and T.R. Seshadri, gr-qc/0007079; N. Banerjee and D. Pavon, Phys. Rev. D 63, 043504 (2001); E. Gunzig, ibid. 63, 067301 (2001).

[13] A. Masiero, M. Pietroni, and F. Rosati, Phys. Rev. D 61, 023504 (2000).

[14] T.R. Taylor and G. Veneziano, Phys. Lett. B 213, 459 (1988).

[15] T. Damour and K. Nordtvedt, Phys. Rev. D 48, 3436 (1993); Phys. Rev. Lett. 70, 2217 (1993); T. Damour and A.M. Polyakov, Nucl. Phys. B423, 532 (1994); Gen. Relativ. Gravit. 26, 1171 (1994).

[16] V. Kaplunovsky, Phys. Rev. Lett. 55, 1036 (1985); M. Dine and N. Seiberg, ibid. 55, 366 (1985); R. Petronzio and G. Veneziano, Mod. Phys. Lett. A 2, 707 (1987).

[17] M.B. Green, J. Schwartz, and E. Witten, Superstring Theory (Cambridge University Press, Cambridge, England, 1987).

[18] E. Witten, Nucl. Phys. B471, 135 (1996).

[19] M. Gasperini, Phys. Rev. D 64, 043510 (2001).

[20] M. Gasperini, Class. Quantum Grav. 17, R1 (2000); G. Veneziano, in Proceedings of the LXXI Les Houches Summer School "The Primordial Universe," edited by P. Binètruy, R. Schaeffer, J. Silk, and F. David (Springer-Verlag, Berlin,
2000), p. 581; M. Gasperini, in Proceedings of the Second SIGRAV School on "Gravitational Waves in Astrophysics, Comology and String Theory, "Centre A. Volta, Como, 1999, edited by I. Ciufolini et al. (IOP Publishing, Bristol, 2001), p. 280; J.E. Lidsey, D. Wands, and E.J. Copeland, Phys. Rep. 337, 343 (2000); an updated collections of papers on the pre-big-bang scenario is also available at http:// www.ba.infn.it/ gasperin

[21] G. Veneziano, in The Geometric Universe, edited by L.J. Mason, K.P. Tod, S.T. Tsou and N.M.J. Woodhouse (Oxford University Press, Oxford, 1998), p. 235; G. Veneziano, "Large-N bounds on, and compositeness limit of, gauge and gravitational interactions," hep-th/0110129.

[22] A.D. Sakharov, Dokl. Akad. Nauk. SSSR 177, 70 (1967) [Sov. Phys. Dokl. 12, 1040 (1968)]; Ya.B. Zel'dovich, Pis'ma Zh. Éksp. Teor. Fiz. 6, 922 (1967) [JETP Lett. 6, 345 (1967)]; D. Amati and G. Veneziano, Phys. Lett. 105B, 358 (1981); Nucl. Phys. B204, 451 (1982), and references therein.

[23] T. Damour, G.W. Gibbons, and C. Gundlach, Phys. Rev. Lett. 64, 123 (1990).

[24] B. Ratra and P.J.E. Peebles, Phys. Rev. D 37, 3406 (1988); I. Zlatev, L. Wang, and P.J. Steinhardt, Phys. Rev. Lett. 82, 896 (1999); Phys. Rev. D 59, 123504 (1999).

[25] M. Gasperini, Phys. Lett. B 470, 67 (1999).

[26] B. Boisseau, G. Esposito-Farese, D. Polarski, and A.A. Starobinsky, Phys. Rev. Lett. 85, 2236 (2000).

[27] P.G. Ferreira and M. Joyce, Phys. Rev. D 58, 023503 (1998).

[28] L. Amendola and D. Tocchini-Valentini, Phys. Rev. D 64, 043509 (2001).

[29] M.S. Turner and A.G. Riess, astro-ph/0106051.

[30] See, for instance, R.W. Hellings, in Gravitational measurements, Fundamental Metrology and Constants, Erice, June, 1987, edited by V.N. Melnikov, Vol. 230 of NATO Advanced Study Institute Series $C$ (Kluwer Acad. Pub., Dordrecht, The Netherlands, 1988), p. 181.

[31] E. Fischbach and C. Talmadge, Nature (London) 356, 207 (1992).

[32] C. Wetterich, Astron. Astrophys. 301, 321 (1995).

[33] L. Amendola, Phys. Rev. D 62, 043511 (2000).

[34] D. J. Holden and D. Wands, Phys. Rev. D 61, 043506 (2000).

[35] W. Zimdahl and D. Pavón, astro-ph/0105479.

[36] L. Wang, R.R. Caldwell, J.P. Ostriker, and P.J. Steinhardt, Astrophys. J. 530, 17 (2000).

[37] A. Balbi, C. Baccigalupi, S. Matarrese, F. Perrotta, and N. Vittorio, Astrophys. J. Lett. 547, L89 (2001).

[38] P. de Bernardis et al., astro-ph/0105296.

[39] M. Doran, M. Lilley, and C. Wetterich, astro-ph/0105457.

[40] T. Barreiro, B. de Carlos, and E.J. Copeland, Phys. Rev. D 58, 083513 (1998).

[41] T. Damour and K. Nordtvedt, Ref. [15]; N. Bartolo and M. Pietroni, Phys. Rev. D 61, 023518 (2000).

[42] T. Damour, in "Proceedings of the Les Houches School, 1992," gr-qc/9606079.

[43] T. Damour Nucl. Phys. B (Proc. Suppl.) 80, 41 (2000).

[44] R. Bean, S.H. Hansen, and A. Melchiorri, astro-ph/0104162.

[45] R. Brustein and P.J. Steinhardt, Phys. Lett. B 302, 196 (1993). 